\title{
Décès liés à l'usage de buprénorphine : investigations toxicologiques dans le cadre de cas médico-légaux
}

\section{Deaths after buprenorphine intake : toxicological investigations in forensic cases}

\section{Vincent CIRIMELE, Sylviane LOHNER, Bertrand LUDES, Pascal KINTZ}

Institut de Médecine Légale, Laboratoire de Toxicologie, 11, rue Humann - F-67085 STRASBOURG - France

Tél : ++33 (0)390243356 - fAX : ++33 (0)390243362 - E-mail : vincent.cirimele@ medecine.strasbg.fr

\section{RÉSUMÉ}

La buprenorphine est utilisée dans le traitement de substitution des pharmacodépendances aux opiacés, et depuis le mois de février 1996, une formulation haut dosage est disponible en France sous la dénomination Subutex®. Depuis cette date, 137 cas d'intoxication mortelle suite à la consommation de buprénorphine ont été décrits. Cet article présente les résultats d'une nouvelle étude rétrospective sur 13 décès imputables à la buprenorphine dans la région de Strasbourg et survenus depuis août 2000. La buprenorphine et la norbuprenorphine ont été dosés dans le sang par HPLC/MS. Les concentrations sanguines de buprénorphine variaient de 0,3 à 7,7 $\mathrm{ng} / \mathrm{mL}$ (moyenne $3,5 \mathrm{ng} / \mathrm{mL}$ ) et de 0,3 to $16,2 \mathrm{ng} / \mathrm{mL}$ (moyenne 2,9 $\mathrm{ng} / \mathrm{mL}$ ) pour son métabolite, la norbuprénorphine. L'injection intraveineuse de buprenorphine après broyage des glossettes, la consommation concomitante de médicaments psychoactifs (benzodiazepines et neuroleptiques) et la formulation haut dosage de buprenorphine disponible en France semblent être déterminants pour l'imputabilité des décès.

\section{MOTS-CLÉS}

Buprenorphine, intoxication, décès, programme de substitution.

\section{SUMMARY}

Buprenorphine at high dosage became available in France in 1996, as a substitution treatment for heroin addicts. Since this date, 137 deaths were attributed to this drug. This paper reports an original serie of 13 fatalities involving buprenorphine observed at the Institute of Legal Medicine of Strasbourg since August 2000. Buprenorphine and its primary metabolite norbuprenorphine were assayed in postmortem blood by HPLC/MS. Blood levels ranged from 0.3 to $7.7 \mathrm{ng} / \mathrm{mL}$ (mean $3.5 \mathrm{ng} / \mathrm{mL}$ ) and from 0.3 to $16.2 \mathrm{ng} / \mathrm{mL}$ (mean $2.9 \mathrm{ng} / \mathrm{mL}$ ) for the parent buprenorphine and its metabolite norbuprenorphine, respectively. Intravenous injection of crushed tablets, the concomitant intake of psychotropics (especially benzodiazepines and neuroleptics) and the high dosage of buprenorphine formulation available in France appear as the major risk factors for such fatalities.

\section{KEY-WORDS}

Buprenorphine, poisoning, fatality, substitution program. 


\section{Introduction}

A faible dose (0,3 à 0,6 mg IV ou IM), la buprenorphine est un puissant analgésique, dont l'activité est 25 à 40 fois plus marquée que celle de la morphine.

Sous la dénomination Temgesic ${ }^{\circledR}$ à la posologie de 0,2 $\mathrm{mg}$, la buprenorphine a été largement prescrite depuis 20 ans pour le traitement des douleurs modérées à sévères, ainsi qu'en anesthésiologie comme prémédication ou comme inducteur.

Plus récemment, la buprenorphine a été reconnue digne d'intérêt comme traitement de substitution dans le traitement des pharmacodépendances aux opiacés. Sous la dénomination Subutex ${ }^{\circledR}$, une présentation haut dosage (glossettes sublinguales à $0,4,2$, et $8 \mathrm{mg}$ ) est disponible en France depuis le mois de février 1996. Contrairement à la méthadone, délivrée quotidiennement dans des centres agrées sur la base d'un suivi strict des toxicomanes, incluant des analyses urinaires hebdomadaires de contrôle, le Subutex ${ }^{\circledR}$ peut être prescrit par tout médecin pour une période de 28 jours au maximum et délivré par tout pharmacien. Les patients n'ont pas obligation de prendre le médicament devant le médecin ou le pharmacien et ne sont généralement pas suivis sur le plan biologique.

La buprenorphine est largement utilisée en France par environ 70 à 80000 personnes dépendantes de l'héroïne, tandis qu'un marché noir parallèle s'avère très actif. Sur le plan sanitaire et social, ce programme de substitution peut être considéré comme un succès, le nombre d'overdoses à l'héroïne ayant chuté de 500 il y a quelques années à moins de 100 pour 1999 tandis qu'on observe une meilleure approche de la prise en charge et de ses bénéfices. Cependant, depuis la première intoxication létale observée par Tracqui et al (1) en août 1996, plusieurs observations similaires ont été faites par des experts judiciaires en France. En 1998, Tracqui et al (2) publiaient une série de 20 décès collectés dans 5 centres. Dans tous les cas, une ingestion concomitante de psychotropes (surtout des benzodiazépines) était notée. Plus récemment, une étude rétrospective plus complète a été publiée sur les décès imputables à la buprenorphine dans la région de Strasbourg de mars 1998 à juillet 2000 et de 13 laboratoires pratiquants des expertises judiciaires en France sur la période mi-1996 à mars 2000 (3).

Ce nouvel article présente les résultats d'une nouvelle étude rétrospective sur les décès imputables à la buprenorphine dans la région de Strasbourg de août 2000 à octobre 2001.

\section{Matériel et méthodes Sujets}

Sur la période étudiée (août 2000 à octobre 2001), l'Institut de Médecine Légale de Strasbourg a pratiqué environ 800 expertises judiciaires en toxicologie dans le cadre des recherches des causes de la mort et a rendu 13 dossiers où de la buprenorphine a été retrouvée dans le sang de la victime et où les décès liés à une cause violente ont été systématiquement écartés. Dans tous les cas, l'autopsie avait mis en évidence des signes d'asphyxie (cyanose, congestion multiviscérale, œdème pulmonaire...). Aucune autre cause de décès n'avait pu être retenue par des médecins légistes expérimentés.

\section{Analyses toxicologiques}

Le dosage de la buprénorphine et de son métabolite a été réalisé par HPLC/MS selon une technique publiée (4) mais modifiée selon le protocole suivant : le sang ( $2 \mathrm{~mL}$ ) additionnés de $15 \mathrm{ng}$ d'étalons deutérés (buprénorphine- $\mathrm{d}_{4}$ et norbuprénorphine- $\mathrm{d}_{3}$ ) est extrait à $\mathrm{pH}$ 9,5 par $10 \mathrm{~mL}$ du mélange chloroforme/2-propanol $/ n$ heptane $(25: 10: 65, \mathrm{v} / \mathrm{v})$. Après agitation horizontale (20 min à 100 cycles/min) et centrifugation (15 min à $3500 \mathrm{rpm}$ ), la phase organique est prélevée et additionnée de $5 \mathrm{~mL}$ d'acide chlorhydrique $0,2 \mathrm{~N}$. Après agitation et centrifugation, la phase acqueuse est prélevée, neutralisée et extraite à $\mathrm{pH} 9,5$ par $5 \mathrm{~mL}$ de chloroforme. La phase organique finale est alors prélevée, évaporée et l'extrait sec repris par $25 \mu \mathrm{L}$ de méthanol.

Pour l'analyse, $5 \mu \mathrm{L}$ sont injectés sur colonne $C_{18}$ Novapak $(150 \times 2,0 \mathrm{~mm})$ traversée par la phase mobile à un débit de $1 \mathrm{~mL} / \mathrm{min}$. La séparation est obtenue à l'aide d'un gradient linéaire d'acétonitrile / tampon $\mathrm{NH}_{4} \mathrm{COOH} 2 \mathrm{mM}$ pH 3,0 (acétonitrine 35 à $70 \%$ en 9 $\min$ ). La détection a été réalisée sur un détecteur de masse API 100 de chez Perkin Elmer Sciex avec une interface de type Ionspray. La tension d'orifice était fixée à $+75 \mathrm{~V}$ et celle du multiplicateur d'électrons à $+2600 \mathrm{~V}$. L'acquisition a été réalisée pour les ions spécifiques des composés recherchés, c'est à dire $m / z 468$ pour la buprénorphine $(\mathrm{m} / z 472$ pour la buprénorphine$\left.\mathrm{d}_{4}\right)$ et $m / z 414$ pour la norbuprénorphine $(\mathrm{m} / z 417$ pour la norbuprénorphine- $\mathrm{d}_{3}$ ).

En complément des analyses spécifiques pour la buprenorphine, un screening très large du sang post mortem a été systématiquement réalisé sur tous les sujets par immunochimie (FPIA) sur l'automate Abbott $\mathrm{ADx}^{\mathrm{TM}}$, spectrophotométrie UV (monoxyde de carbone), GC/FID (méprobamate, éthanol), head-space GC/NPD (cyanures), head-space GC/MS (substances volatiles) et LC/DAD + GC/MS (médicaments, stupéfiants, produits agricoles). 


\section{Résultats et discussion}

L'usage en pratique médico-légale veut que l'expert compare ses résultats avec les données de la littérature à partir de concentrations dites thérapeutiques, toxiques et mortelles. Dans le cas de la buprenorphine, ces données font largement défaut. Quelques études contrôlées donnent des concentrations plasmatiques thérapeutiques de l'ordre de 2 à $20 \mathrm{ng} / \mathrm{mL}$ (5). Après une dose sublinguale unique de $0,4 \mathrm{mg}$, Bullingham et al rapportent des concentrations plasmatiques de l'ordre de 0,45 à $0,84 \mathrm{ng} / \mathrm{mL}$ (6). Selon Kuhlman et al (7), les concentrations plasmatiques moyennes de buprenorphine chez 6 sujets recevant $4,0 \mathrm{mg}$ en sublingual ou par voie orale était de l'ordre de $3,31 \mathrm{ng} / \mathrm{mL}(1,93$ à $7,19 \mathrm{ng} / \mathrm{mL})$ et de $1,98 \mathrm{ng} / \mathrm{mL}(0,25$ à $3,90 \mathrm{ng} / \mathrm{mL})$. Aucune concentration toxique ne semble avoir été établie et c'est ainsi que Tracqui et al (2) ont attribué à la buprenorphine 20 décès, même à concentration thérapeutique, en absence de toute autre cause probante. Ces auteurs ont conclu que la buprenorphine pouvait être responsable d'une dépression respiratoire potentiellement létale lorsque associée à d'autres psychotropes, alors même que tous les xénobiotiques étaient présents à des concentrations compatibles avec la thérapeutique. De nombreuses expertises judiciaires récentes, pratiquées à Strasbourg et dans d'autres centres français ont confirmé ces résultats préliminaires. Les données toxicologiques de ces décès sont rapportés dans le Tableau 1 (3).

Pour la période étudiée (août 2000 - octobre 2001), 13 nouveaux cas de décès imputables à la buprenorphine dans la région de Strasbourg ont été observés. Les concentrations sanguines de buprénorphine ont varié de 0,3 à $7,7 \mathrm{ng} / \mathrm{mL}$ (moyenne $3,5 \mathrm{ng} / \mathrm{mL}$ ) et de 0,3 to
$16,2 \mathrm{ng} / \mathrm{mL}$ (moyenne $2,9 \mathrm{ng} / \mathrm{mL}$ ) pour son métabolite, la norbuprénorphine.

Les circonstances des décès ont suggéré dans la grande majorité des cas une polyintoxication dans un contexte d'usage de buprenorphine : boîtes vides de Subutex ${ }^{\circledR}$ et/ou présence de buprenorphine dans du matériel (cuillère, seringue...), autres psychotropes (médicaments, stupéfiants). Des signes de violence n'ont jamais été retrouvés lors de l'autopsie, mais tous les corps présentaient les marques d'une asphyxie prolongée, classique pour tout surdosage par dépresseurs du système nerveux central, en particulier pour les narcotiques. Des traces d'injection récentes ont été remarquées dans $3 / 4$ des cas.

La totalité des résultats concernant ces 13 nouveaux cas d'intoxication mortelle par buprénorphine observés à l'Institut de Médecine Légale de Strasbourg pendant la période août 2000 - octobre 2001 sont détaillés dans le Tableau 2.

Les associations buprenorphine-benzodiazepines sont très fréquentes, et sont retrouvées dans 9 observations. La potentialisation des effets dépresseurs de la buprenorphine par les benzodiazépines a déjà été notée dans plusieurs observations cliniques, chez des patients anesthésiés (8). De telles interactions doivent également avoir lieu avec d'autres classes pharmacologiques, comme les neuroleptiques ou les antidépresseurs. La cyamemazine était présente dans 2 cas, l'alimémazine et l'amitryptiline dans un cas. Un usage concomitant de stupéfiants a été observé dans 8 cas, avec majoritairement 6 cas pour le cannabis et 3 cas pour la cocaine. Les derniers cas cités avec abus de cocaine ou de solvants sont les premiers recensés dans la région strasbourgeoise.

Tableau I : Données toxicologiques pour 117 décès observés de mi-1996 à juillet 2000 (selon ref. 3).

\begin{tabular}{|c|c|c|}
\hline & Strasbourg $(\mathbf{n}=39)$ & 13 autres centres $(\mathbf{n}=\mathbf{7 8})$ \\
\hline Concentrations en buprénorphine dans le sang & 0,5 à $51,0 \mathrm{ng} / \mathrm{mL}($ moyenne $10,2 \mathrm{ng} / \mathrm{mL})$ & 0,1 à $76,0 \mathrm{ng} / \mathrm{mL}(\mathrm{moyenne} 12,6 \mathrm{ng} / \mathrm{mL})$ \\
\hline Concentrations en norbuprénorphine dans le sang & 0,2 à $47,1 \mathrm{ng} / \mathrm{mL}($ moyenne $8,2 \mathrm{ng} / \mathrm{mL})$ & $<0,1$ à $65,0 \mathrm{ng} / \mathrm{mL}(\mathrm{moyenne} 10,6 \mathrm{ng} / \mathrm{mL})$ \\
\hline Buprénorphine + éthanol + divers & $10 \mathrm{cas}(25,6 \%)$ & $24 \mathrm{cas}(30,8 \%)$ \\
\hline Buprénorphine + benzodiazépines & 31 cas $(79,5 \%)$ & $60 \mathrm{cas}(76,9 \%)$ \\
\hline Buprénorphine + neuroleptiques & $18 \mathrm{cas}(46,2 \%)$ & 19 cas $(24,3 \%)$ \\
\hline Buprénorphine + autres psychotropes & 8 cas $(20,5 \%)$ & 16 cas $(20,5 \%)$ \\
\hline Buprénorphine + narcotiques & 3 cas $(7,6 \%)$ & 20 cas $(25,6 \%)$ \\
\hline Buprénorphine + cocaine & 0 cas & 6 cas $(7,7 \%)$ \\
\hline Buprénorphine + cannabis & 22 cas $(56,4 \%)$ & 36 cas $(46,2 \%)$ \\
\hline
\end{tabular}




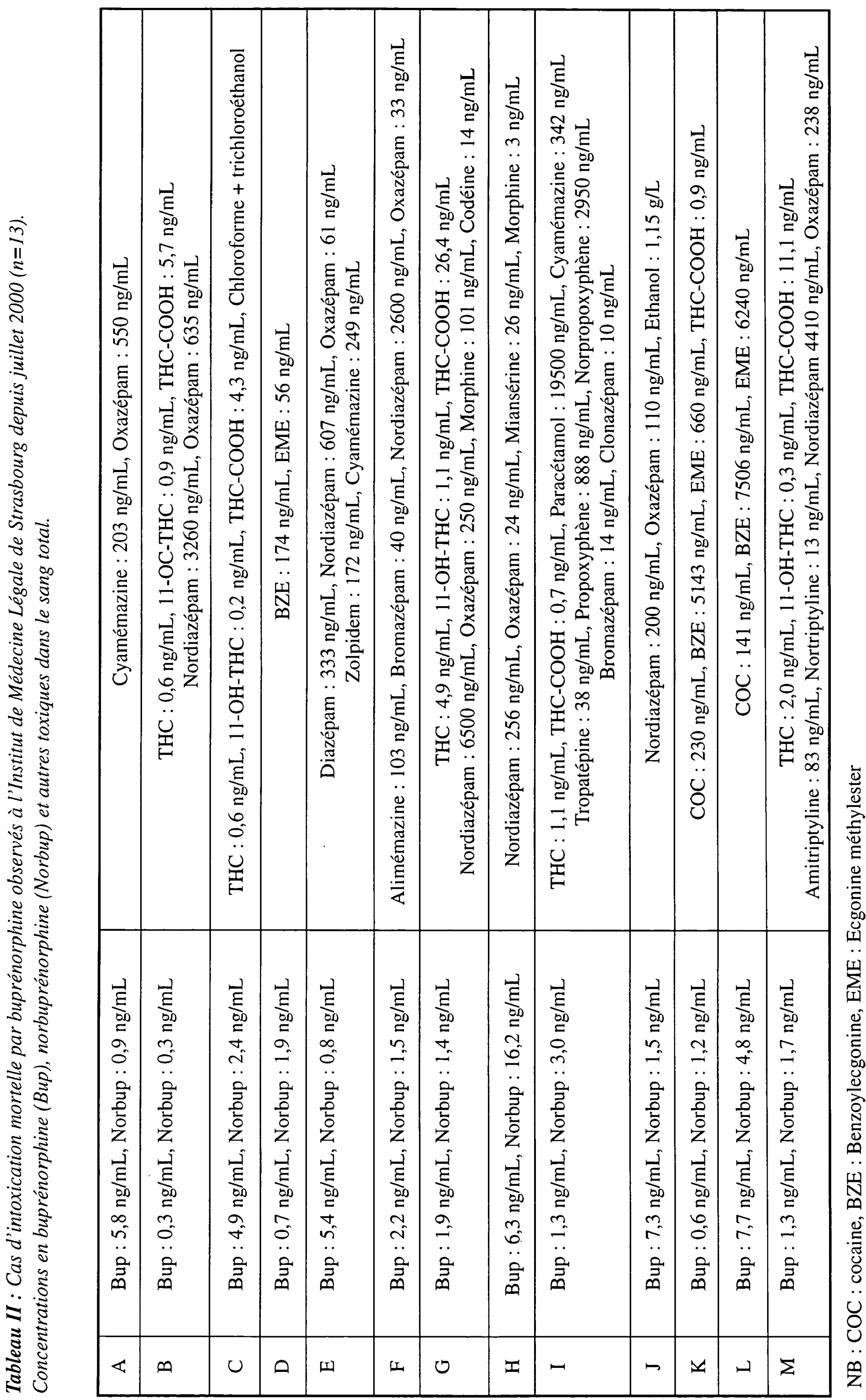


L'injection intraveineuse de buprenorphine après broyage des glossettes constitue très certainement un autre facteur de risque, la plupart des dépressions respiratoires publiées après usage médical se rapportant à une administration IV (9). Ce type d'administration conduit à une saturation quasi-instantanée des récepteurs centraux aux opiacés et à une bio-disponibilité maximale de la buprenorphine qui est généralement faible, en particulier per os (20-30\%).

Enfin, la concentration de principe actif sous forme de buprenorphine haut dosage dans le Subutex ${ }^{\circledR}$ pourrait jouer un rôle dans ces accidents, malgré un effet plafond théorique, en relation avec l'activité pharmacodynamique double (agoniste/antagoniste) présenté souvent comme sensé réduire les risques (10).

\section{Conclusion}

En résumé, à ce jour, les décès par association buprenorphine haut dosage et psychotrope(s) semblent être un problème exclusivement français, car aucun autre cas n'a été publié dans la littérature. Les risques encourus par les toxicomanes sembles être majorés par l'usage concomitant de psychotropes, surtout les benzodiazépines et les neuroleptiques, et l'injection intraveineuse. Le nombre de décès imputables à cette dangereuse association est très certainement sous-estimé du fait de la difficulté analytique à caractériser la buprenorphine (faible concentration circulante, pas de méthode simple d'immunochimie pour le sang).

\section{Références}

1. Tracqui A., Petit G., Potard D., Lévy F., Kintz P., Ludes B. Intoxications mortelles par buprénorphine (Subutex) et benzodiazépines : 4 cas. J. Med. Leg. Droit Méd. 1997 ; $40: 213-23$.

2. Tracqui A., Kintz P., Ludes B. Buprenorphine-related deaths among drug addicts in France : a report on 20 fatalities. J. Anal. Toxicol. $1998 ; 22$ : 430-4.

3. Kintz P. Deaths involving buprenorphine : a compendium of French cases. Forensic Sci. Int. $2001 ; 121$; 65-9.

4. Tracqui A., Kintz P., Mangin P. HPLC/MS determination of buprenorphine and norbuprenorphine in biological fluids and hair samples. J. Forensic Sci. 1997 ; 42 : 111 4.

5. Huestis M. Controlled buprenorphine administration studies, workshop on Pharmacology and toxicology of buprenorphine, 52nd annual meeting of the American Academy of Forensic Science, February 21-26, 2000, Reno, NV.
6. Bullingham R.E.S., McQuay H.J., Porter E.J.B., Allen M.C., Moore R.A. Sublingual buprenorphine used postoperatively: Ten hour plasma drug concentration analysis. Br. J. Clin. Pharmacol. 1982; 13 : 665-73.

7. Kuhlman J.J., Lalani S., Magluilo J., Levine B., Darwin W.D., Johnson R.E., Cone E.J. Human pharmacokinetics of intravenous, sublingual, and buccal buprenorphine. J. Anal. Toxicol. $1996 ; 20: 369-78$.

8. Kay B. Buprenorphine, benzodiazepines and respiratory depression. Anaesthesia $1984 ; 39: 491-2$.

9. Downing J.W., Goodwin N.M., Hicks J. The respiratory depressive effects of intravenous buprenorphine in patients in an intensive care unit. S. Afr. Med. J. 1979 ; $55: 1023-7$.

10. Walsh S.L., Preston K.L., Stitzer M.L., Cone E.J., Bigelow G.E. Clinical pharmacology of buprenorphine: ceiling effects at high doses. Clin. Pharmacol. Ther. $1994 ; 55: 569-80$. 